\title{
Bendable and twistable crystals of flufenamic acid form III with
} bending mechanofluorochromism behavior

Yu Liu ${ }^{1}$, Peng Yang ${ }^{1}$, Keke Zhang ${ }^{1}$, Jun Xu ${ }^{2}$, Songgu Wu ${ }^{1 *} \&$ Junbo Gong ${ }^{1}$

1. State Key Laboratory of Chemical Engineering, Tianjin University, Tianjin 300072, P. R. China.

2. Health Science Platform, Tianjin University, Tianjin 300072, P. R. China

\section{Supporting information}

\section{Table of contents}

Figure S1. Face indexing results of FFA form III crystal.

Figure S2. Single crystals of FFA form III in different sizes.

Figure S3. Nanoindentation data of FFA form III crystal.

Figure S4. Hydrogen bonds and aromatic interactions in FFA form III crystal.

Figure S5. Energy frameworks of FFA form III crystal

Figure S6. Molecular structure pairs and the interaction energies obtained from energy frameworks.

Figure S7. Intermolecular interactions in FFA form I crystal.

Figure S8. Crystal packing patterns for FFA form I crystal.

Table S1. Crystallographic data for FFA form III crystals.

Table S2. The changes of intermolecular interactions in bent crystals. 


\section{Face indexing results}

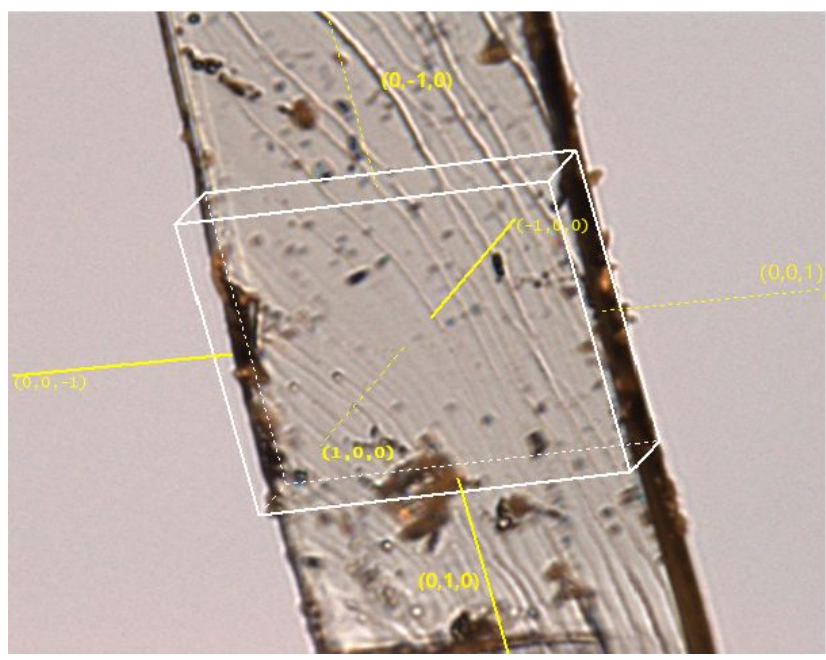

Figure S1. Face indexing of FFA form III crystal.
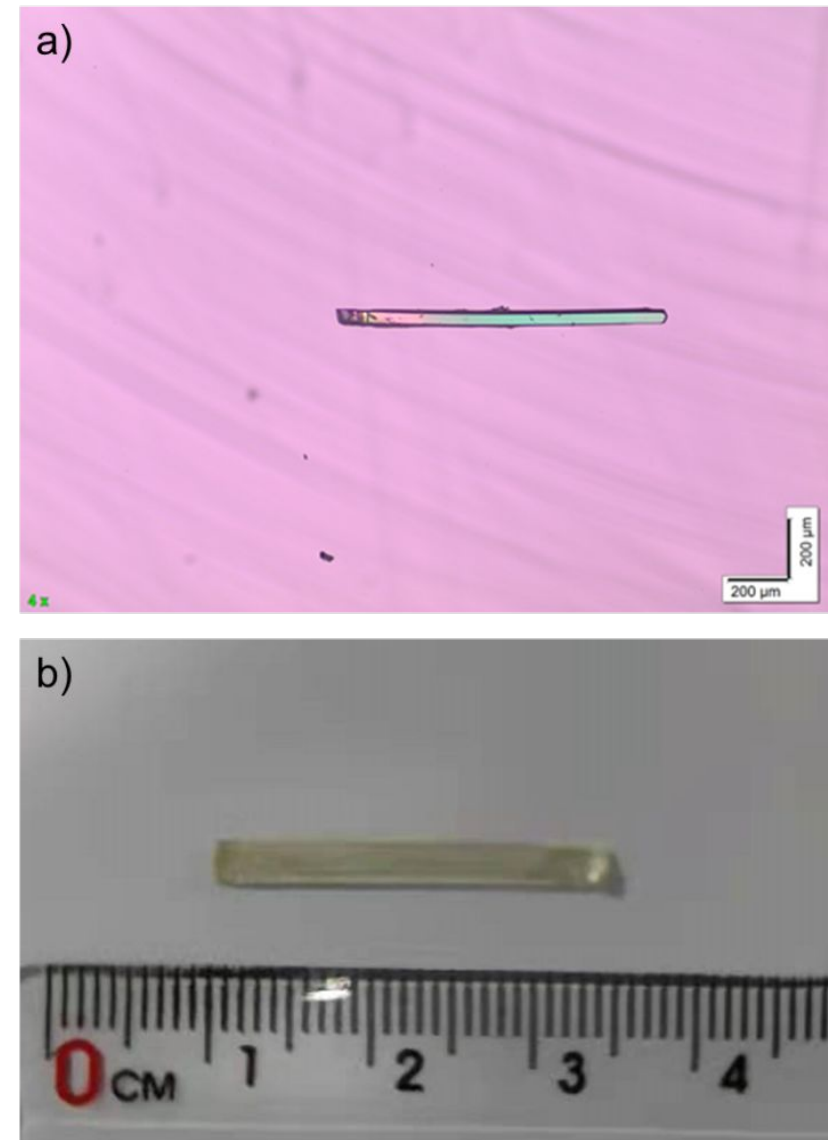

Figure S2. Single crystals of FFA form III in different size. (a) Single crystal in small size. (b) Single crystal in large size. 


\section{Nanoindentation}

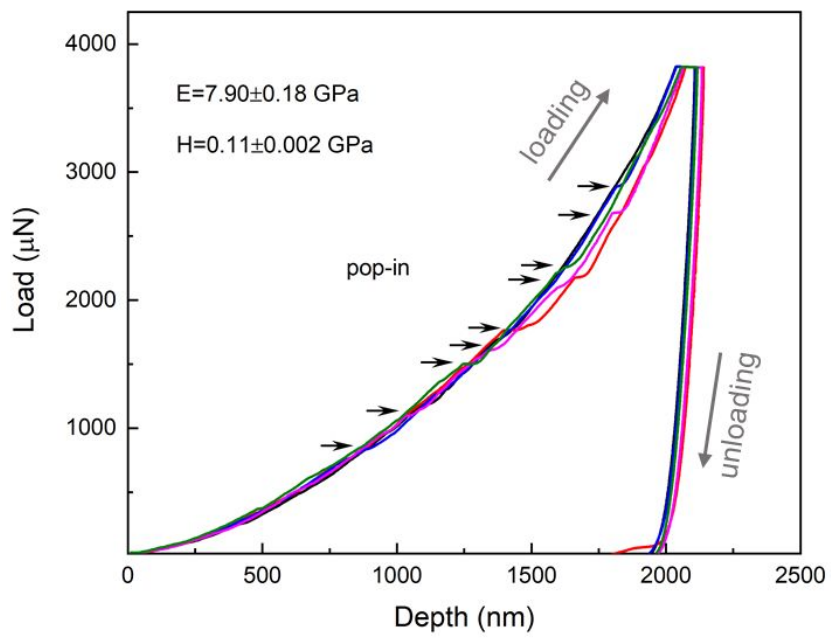

Figure S3. Nanoindentation data from the FFA form III crystals. Load - depth curves at indentation loads of $4 \mathrm{mN}$ on the (100) face of FFA form III single crystal. The arrows pointing to the right indicate the pop-ins in the loading segment.

\section{Intermolecular interactions}

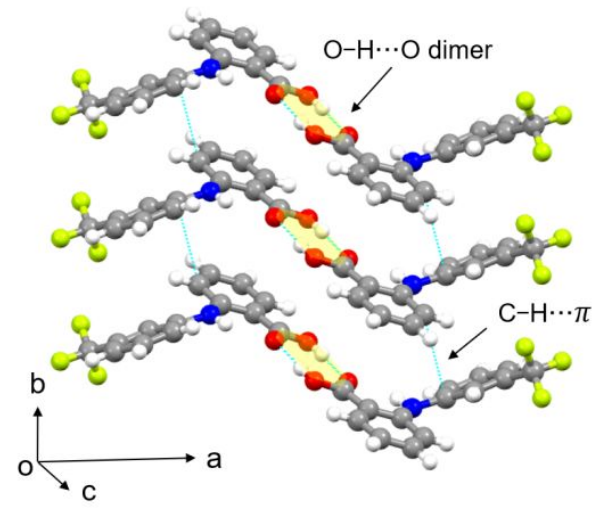

Figure S4. Hydrogen bonds and aromatic interactions in FFA form III crystal, forming stable columns along $b$ axis. 


\section{Energy framework analysis}

CrystalExplorer $17^{1,2}$ was used to evaluate and visualize the pairwise interaction energies of FFA crystal. The calculations are based on the B3LYP/6-31G(d.p) molecular wavefunctions. The energy components calculated within this method are electrostatic, polarization, dispersion, and exchange-repulsion and finally the total interaction energy, where E_total $=1.057 \mathrm{E} \_$ele + $0.740 \mathrm{E} \_$pol $+0.871 \mathrm{E} \_$disp +0.618 E_rep. Pairwise interaction energies between molecules were calculated considering radius of $3.8 \AA$ from centroid of a molecule to an atom (of another molecule) belonging to its nearest neighbor. The tube size used in all the energy frameworks was 150 , and the lower energy threshold (cutoff) value was set to 0 .

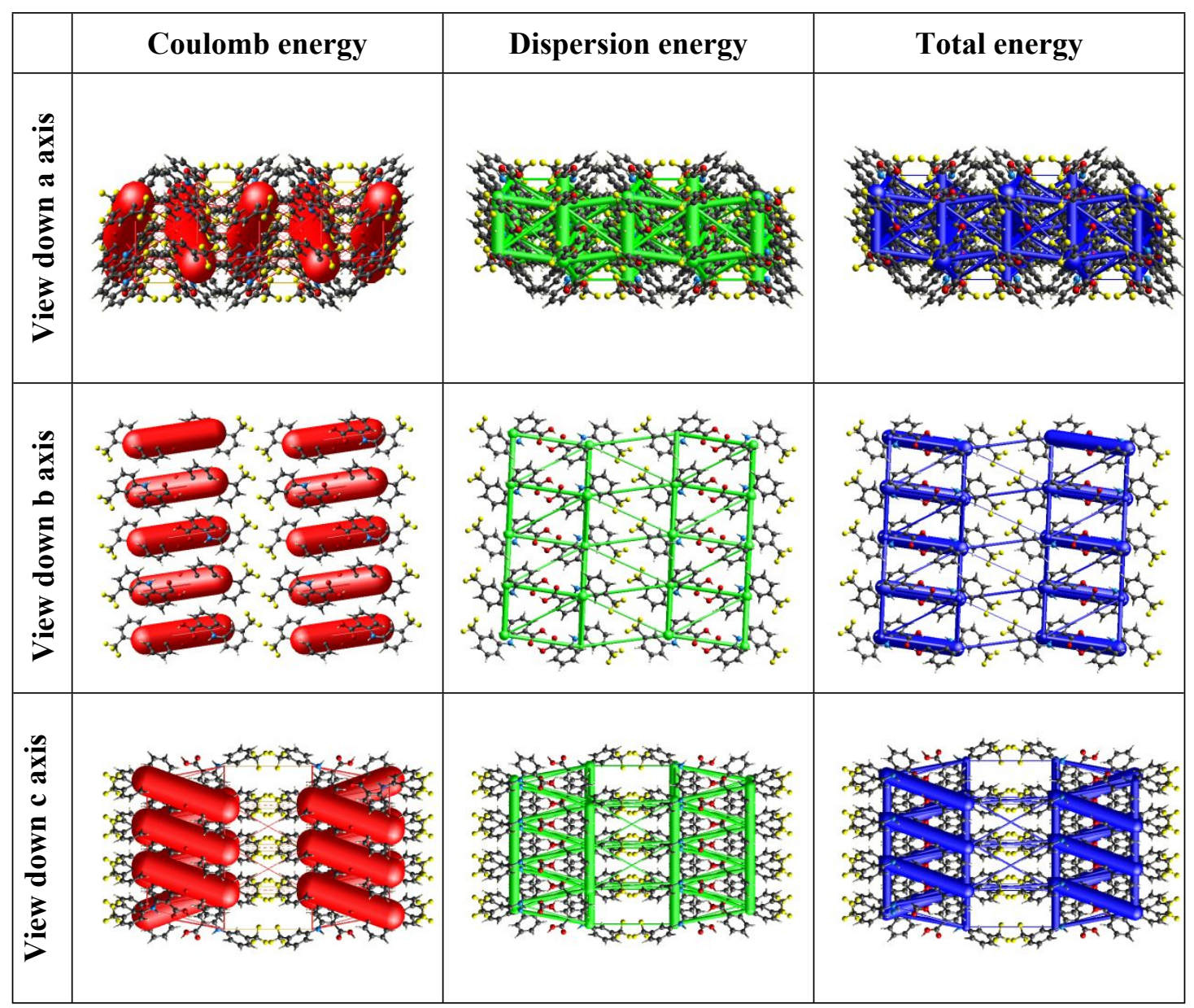

Figure S5. Energy frameworks of FFA viewed along different crystallographic directions. 


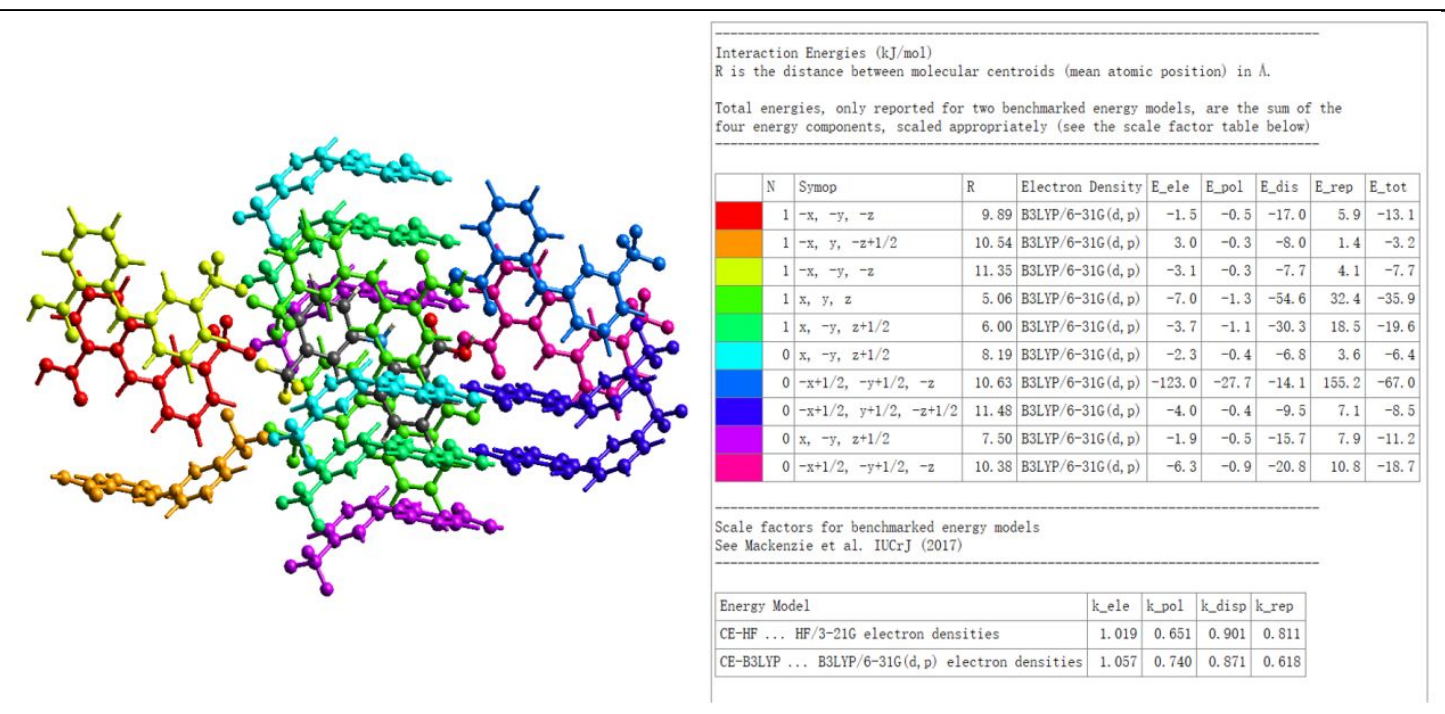

Figure S6. Molecular structure pairs and the interaction energies $(\mathrm{kJ} / \mathrm{mole})$ obtained from energy frameworks calculation for FFA.

\section{Crystal structure analysis of FFA form I}

Hydrogen-bond dimer

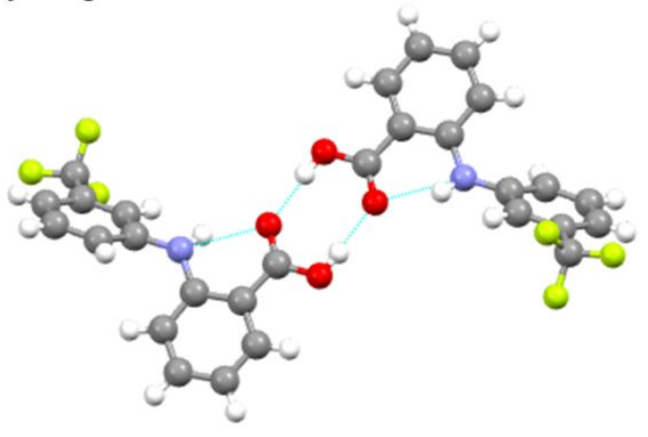

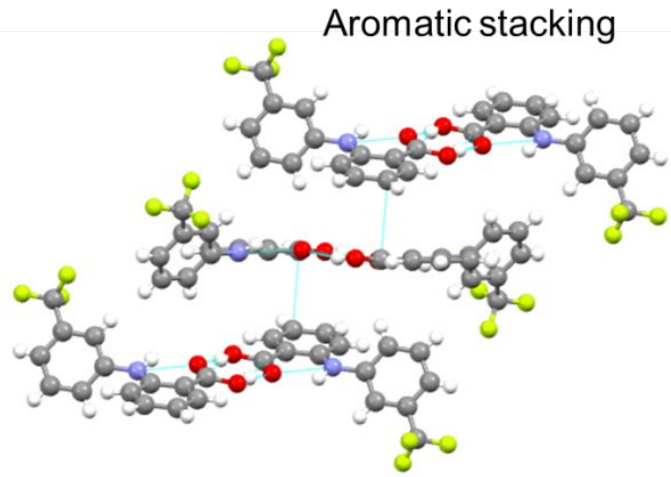

Figure S7. Intermolecular interactions in FFA form I crystal.

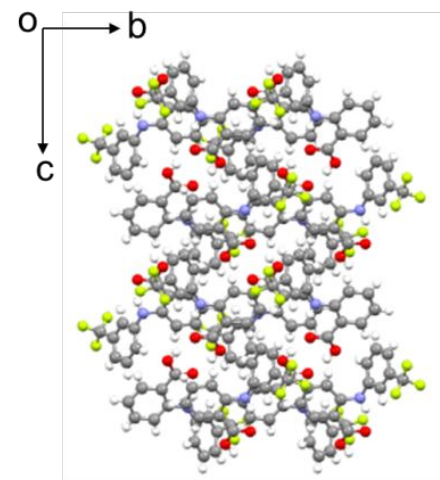

a

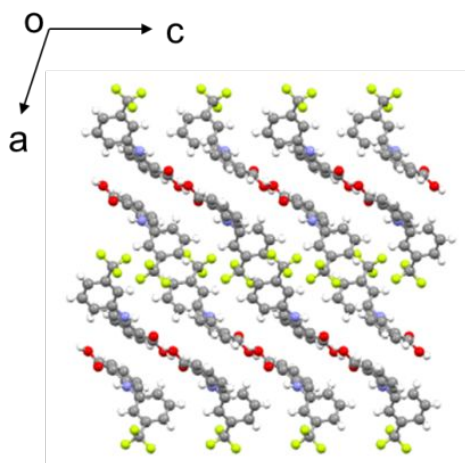

b

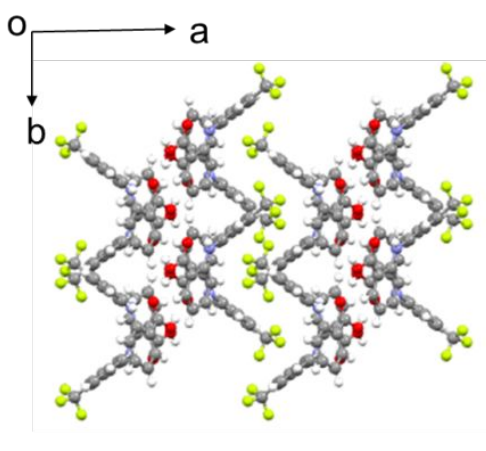

C

Figure S8. Crystal packing patterns for FFA form I viewed along a-axis, b-axis and c axis. 


\section{Single crystal structure results}

Table S1. Crystallographic data for FFA form III crystals.

\begin{tabular}{|c|c|c|}
\hline & Straight & Bent \\
\hline Empirical formula & $\mathrm{C}_{14} \mathrm{H}_{10} \mathrm{~F}_{3} \mathrm{NO}_{2}$ & $\mathrm{C}_{14} \mathrm{H}_{10} \mathrm{~F}_{3} \mathrm{NO}_{2}$ \\
\hline Temperature (K) & $106(2)$ & $106(2)$ \\
\hline Crystal system & Monoclinic & Monoclinic \\
\hline Space group & $C 2 / c$ & $C 2 / c$ \\
\hline $\mathrm{a}(\AA)$ & $39.6616(6)$ & $39.6836(17)$ \\
\hline $\mathrm{b}(\AA)$ & $5.05610(10)$ & $5.0550(2)$ \\
\hline$c(\AA)$ & $11.9613(2)$ & $11.9667(5)$ \\
\hline$\alpha\left(^{\circ}\right)$ & 90 & 90 \\
\hline$\beta\left(^{\circ}\right)$ & $91.962(2)$ & $91.939(4)$ \\
\hline$\gamma\left({ }^{\circ}\right)$ & 90 & 90 \\
\hline$V\left(A^{-3}\right)$ & $2397.23(7)$ & $2399.15(17)$ \\
\hline $\mathrm{Z}$ & 8 & 8 \\
\hline$\rho_{\text {calc }}\left(\right.$ g.cm $\left.\mathrm{cm}^{-3}\right)$ & 1.558 & 1.557 \\
\hline$\theta$ range & $3.6830-72.9580$ & $2.218-73.775$ \\
\hline \multirow[t]{3}{*}{ Index ranges } & $-49 \leq \mathrm{h} \leq 47$ & $-49 \leq \mathrm{h} \leq 48$ \\
\hline & $-6 \leq \mathrm{k} \leq 5$ & $-2 \leq \mathrm{k} \leq 6$ \\
\hline & $-14 \leq 1 \leq 14$ & $-14 \leq 1 \leq 14$ \\
\hline Reflections collected & 19142 & 6976 \\
\hline $\mathrm{R}_{\text {int }}$ & 0.0278 & 0.0638 \\
\hline Final $\mathrm{R}$ indexes $[\mathrm{I}>=2 \sigma(\mathrm{I})]$ & 0.0375 & 0.1164 \\
\hline $\mathrm{wR}_{2}$ & 0.1029 & 0.3294 \\
\hline Godness-of-fit & 1.066 & 1.623 \\
\hline CCDC number & 2034097 & 2034319 \\
\hline
\end{tabular}

Table S2. Intermolecular interactions within the structure responsible for bending in FFA and other shortest non-bonding distances between the molecules.

\begin{tabular}{|l|c|c|c|c|}
\hline & $\mathrm{d}(\mathrm{H} \cdots \mathrm{O})(\AA)$ & $\angle \mathrm{O}-\mathrm{H} \cdots \mathrm{O}\left({ }^{\circ}\right)$ & $\mathrm{d}(\mathrm{C}-\mathrm{H} \cdots \pi)(\AA)$ & $\begin{array}{c}\text { Closest } \mathrm{F} \cdots \mathrm{F} \\
\operatorname{contact}(\AA)\end{array}$ \\
\hline Straight crystal & $1.750(30)$ & $176(2)$ & $2.7767(7)$ & $3.0379(14)$ \\
\hline Bent crystal & $1.812(2)$ & $173.76(17)$ & $2.7770(18)$ & $3.042(3)$ \\
\hline
\end{tabular}




\section{Notes and references}

(1) M. J. Turner, S. Grabowsky, D. Jayatilaka and M. A. Spackman, J. Phys. Chem. Lett., 2014, 5, 4249-4255.

(2) M. J. Turner, S. P. Thomas, M. W. Shi, D. Jayatilaka and M. A. Spackman, Chem. Comm., 2015, 51, 3735-3738. 